\title{
EDITORIAL
}

\section{In this Issue: Continuity of Care}

\author{
Kurt C. Stange, $M D, P b D$, Editor \\ Departments of Family Medicine, Epidemiology \& Biostatistics, and Sociology, \\ and the Comprehensive Cancer Center, Case Western Reserve University, Cleveland, Ohio \\ Ann Fam Med 2003;1:130-131. DOI: 10.1370/afm.85.
}

"A sustained partnership between patients and clinicians $^{\prime \prime}$ is held by the Institute of Medicine (IOM) to be a critical element of primary care. This sustained partnership is commonly called continuity of care. It is a cornerstone for realizing other aspects of primary care, as defined by the IOM, including integration of care, accountability for a large majority of personal health care needs, and practicing in the context of family and community.

The ability of patients and clinicians to achieve this sustained partnership is under attack. Health care system changes are resulting in forced discontinuity of care. ${ }^{2,3}$ This disruption differentially affects vulnerable patients. ${ }^{4}$ If the trend is not reversed soon, a generation of patients and clinicians will live without the everyday experience of longitudinal, trusting, healing relationships. Not knowing the possibilities inherent in these relationships, we will not make the best decisions about individual health care or systems redesign. ${ }^{5}$

It is therefore timely that a cluster of early manuscripts submitted to the Annals focused on important questions about continuity of care. Continuity is important to clinicians, but does it matter to patients? If so, which patients and when? ${ }^{6}$ Does continuity affect important health care outcomes, such as the quality of diabetes care? ${ }^{7}$ Does it matter for health care costs $^{8,9}$ or utilization? ${ }^{9}$ What are the important dimensions of continuity in the interpersonal healing relationship, and how can these be measured to advance understanding? ${ }^{10}$ Is continuity merely part of the process of care, or do we know enough about its effects to consider it to be an important outcome? ${ }^{11}$

Research articles by Nutting and colleagues, ${ }^{6}{ }^{6}$ Gill et $\mathrm{al}_{1}{ }^{7}$ Saultz, ${ }^{10}$ Franks et $\mathrm{al},{ }^{9}$ and De Maeseneer et $\mathrm{al}_{,}{ }^{8}$ and an editorial by Christakis ${ }^{11}$ tackle these and related questions. Together, they sharpen our focus on what is important about continuity, for whom, and in what situations. They point to the need to pay attention to the crisis in continuity and the lack of systems support for healing relationships. ${ }^{5}$ They point the way toward future research on this foundational topic. We encourage readers who have experience with continuity or its absence to take part in the online discussion of these articles at www.annfammed. org. The diverse perspectives of patients, clinicians, and policymakers are important in fully exploring this important issue.

We encourage readers to participate also in the discussion of the other research papers in this issue. The study by Sax and Kautz ${ }^{12}$ finds that teachers are the most common source of referral for consideration of the diagnosis of attention-deficit/hyperactivity disorder. Knowing the source of referral might have important implications for identifying children with ADHD and preventing overdiagnosis. The essay by Frey ${ }^{13}$ shares the hopeful story of a young immigrant. It gives us a flavor of what a culturally attentive clinician can appreciate and what a supportive social context can achieve.

Readers continue to share important insights in TRACK, the Annals online discussion of articles. In this issue's On-TRACK, Senior Associate Editor William Phillips identifies several interesting themes from recent discussions. We value your continued participation.

To read commentaries or to post a response to this article, see the online version at http://annfammed/cgi/content/full/1/3/130.

\section{References}

1. Donaldson MS, Yordy KD, Lohr KN, Vanselow NA, eds. Primary care: America's health in a new era. Washington DC: National Academy Press; 1996:3.

2. Davis K, Collins KS, Schoen C, Morris C. Choice matters: Enrollees' views of their health plans. Health Aff (Millwood) 1995;14:99-112.

3. Flocke SA, Stange KC, Zyzanski SJ. The impact of insurance type and forced discontinuity on the delivery of primary care. J Fam Pract 1997;45:129-135.

4. Kahana E, Stange KC, Meehan R, Raff L. Forced disruption in continuity of primary care: the patients' perspective. Sociol Focus 1997;30:172-182.

5. Institute of Medicine. Crossing the Quality Chasm: A New Health System for the 21st Century. Washington, DC: National Academy Press; 2001.

6. Nutting PA, Goodwin MA, Flocke SA, Zyzanski SJ, Stange KC. Continuity of primary care: to whom does it matter an when? Ann Fam Med 2003;1:149-155. 
7. Gill JM, Mainous AG III, Diamond JJ, Lenhard MJ. Impact of provider continuity on quality of care for persons with diabetes mellitus. Ann Fam Med 2003;1:162-170.

8. De Maeseneer JM, De Prins L, Gosset C, Heyerick J. Provider continuity in family medicine: does it make a difference for total health care costs? Ann Fam Med 2003;1:144-148.

9. Franks P, Cameron C, Bertakis KD. On being new to an insurance plan: health care use associated with the first years in a health insurance plan. Ann Fam Med 2003;1:156-161.
10. Saultz JW. Defining and measuring interpersonal continuity of care. Ann Fam Med 2003:1:134-143.

11. Christakis DA. Continuity of care: process or outcome? Ann Fam Med 2003;1:131-133.

12. Sax L, Kautz KJ. Who first suggests the diagnosis of attentiondeficit/hyperactivity disorder? Ann Fam Med 2003;1:171-174.

13. Frey JJ 3rd. A journey to someplace better. Ann Fam Med 2003;1: 175-176.

\section{EDITORIAL}

\section{Continuity of Care: Process or Outcome?}

Dimitri A. Christakis, MD, MPH

Child Health Institute, University of Washington, Seattle, Wash

Ann Fam Med 2003:1:131-133. DOI: 10.1370/afm.86.

$\mathrm{P}$ erhaps nothing has been deemed more central to the salubrious patient-physician relationship than continuity of care: it is a core component of the Institute of Medicine's definition of primary care. ${ }^{1}$ Having a regular physician seems vital to the establishment of trust and is frequently lamented as belonging to a bygone era when solo practitioners predominated. ${ }^{2}$ Given such strong face validity, as well as the endorsement of professional societies, ${ }^{3}$ one might ask why the value of continuity need be proved. Why subject something as fundamental as consistent contact with a clinician to the scrutiny of the evidence-based medicine movement? Why not simply take it as an unassailably desirable thing?

For true skeptics, of course, nothing is to be exempted from rigorous study. ${ }^{4}$ Even for those who believe that continuity is inherently good, there are reasons to assess its potential effects, as authors in this issue of Annals of Family Medicine have done. Previous studies of continuity of care have led to conflicting conclusions as to its value. ${ }^{5-12}$ Furthermore, many

Conflict of interest: none reported

\section{CORRESPONDING AUTHOR}

Dimitri A. Christakis, MD, MPH

$6200 \mathrm{NE} 74^{\text {th }}$ St. Suite 210

Seattle WA 98115

dachris@u.washington.edu changes in care delivery arising in response to the increasingly competitive medical market place might potentially diminish continuity of care. The larger size of physician groups, the increasing use of physician extenders, and the shifting alliances of health plans with clinicians, all might hamper patients' or clinicians' attempts to establish and maintain consistent contact. ${ }^{2,13}$ Evaluating the effects of continuity of care might therefore be timely and necessary to countervail forces that could otherwise undermine it. So what more have we learned about continuity of care as a result of the studies in this issue?

Gill et a ${ }^{14}$ fail to find an association between continuity of care and some well-established process measures for high-quality care in diabetic patients. Although their findings appear to conflict with those of another similar study ${ }_{1}{ }^{15}$ there are some important distinctions. The general continuity of care achieved in their sample was quite good. A Continuity of Care index rating of .51 is considerably higher than what others have found in publically insured populations and even higher than was achieved in a randomized trial of continuity of care. ${ }^{5,6}$ Second, the overall quality of care, at least with respect to regular measurement of glycosylated hemoglobin levels, was quite good-an annual screening rate of $81 \%$ might rightly be envied by many medical directors. The high levels of general continuity of care and overall quality of care might combine to create a ceiling effect, which means that 